JouRnal OF Universal Mathematics

Vol.1 No.2 PP.130-147 (2018)

ISSN-2618-5660

\title{
EXISTENCE OF MILD SOLUTIONS FOR AN IMPULSIVE FRACTIONAL INTEGRO-DIFFERENTIAL EQUATIONS WITH NON-LOCAL CONDITION
}

\author{
K. HILAL, L. IBNELAZYZ, K. GUIDA, AND S. MELLIANI
}

\begin{abstract}
In this paper we are interested in studying the existence of solutions for a controlled impulsive fractional evolution equations. We use several tools such as fractional calculus, fixed point theorems and the theory of semigroup. We first give some preliminaries and notations, the second part of the work we provide an existence result for our problem and in the final section, we give some examples to show the importance of our results.
\end{abstract}

\section{INTRODUCTION}

The theory of non-integer calculus has been introduced in 1695 by Leibniz and L'Hopital. Fractional calculus caracterize the memory in the evolution process and it is an important tool in describing real-world phenomena and it is used in many fields, such as physics, biology and economics.

Fractional differential equations with instanteneous impulses have been introduced as a new exciting and interesting branch. But, this type of equations cannot describe the dynamics of evolution process in many areas of research, such as pharmacotherapy. That is why, Hernandez and O'Regan [5] introduced a new model which is the impulsive differential equations with non-instantaneous impulses.

In [8] the authors studied the following non-instantaneous model

$$
\left\{\begin{array}{l}
{ }^{c} D^{\alpha} x(t)=A x(t)+f(t, x(t))+B(t) u(t), t \in \cup_{i=0}^{N}\left(s_{i}, t_{i+1}\right], u \in U_{a d} \\
x(t)=g_{i}(t, x(t)), t \in\left(t_{i}, s_{i}\right], i=1,2, \ldots, N \\
x\left(s_{i}^{+}\right)=x\left(s_{i}^{-}\right), i=1,2, \ldots, N \\
x(0)=x_{0} \in X
\end{array}\right.
$$

Where ${ }^{c} D^{\alpha}$ denotes the Caputo fractional derivatives of order $\alpha \in(0,1)$ with the lower limit zero,

$A: D(A) \subset X \longrightarrow X$ is the generator of a $C_{0}$-semigroup of bounded operators

Date: July 1, 2018, accepted.

Key words and phrases. Fractional, Impulsive differential equations, Krasnoselskii fixed point theorem. 
$T(t)_{t>0}$ on a Banach space $(X,\|\cdot\|), x_{0} \in X, 0=t_{0}=s_{0}<t_{1}<s_{1}<t_{2}<s_{2}<$ $\ldots<t_{m} \leq s_{m}<t_{m+1}=T$ are fixed numbers, $g_{i} \in C(J \times X ; X)$.

The symbols $x\left(s_{i}^{+}\right)$and $x\left(s_{i}^{-}\right)$represents the limits of $x(t)$ at $t=s_{i}$.

Motivated by the work of S. Liu and J. Wang in [8], we study the impulsive differential equation

$$
\left\{\begin{array}{l}
{ }^{c} D^{\alpha} x(t)=A x(t)+f(t, x(t), F x(t), B x(t))+\int_{0}^{t} q(t-s) k(s, x(s)) d s+ \\
C(t) u(t), t \in\left(s_{i}, t_{i+1}\right], i=0,1,2, \ldots, m, u \in U_{a d} \\
{ }^{c} D^{\beta} x(t)=g_{i}(t, x(t)), t \in\left(t_{i}, s_{i}\right], i=1,2, \ldots, m \\
x(0)=x_{0}+h(x) .
\end{array}\right.
$$

Where ${ }^{c} D^{\alpha},{ }^{c} D^{\beta}$ are the Caputo fractional derivatives of order $\alpha \in(0,1)$ and $\beta \in$ $(0,1)$ respectively with the lower limit zero, $A: D(A) \subset X \longrightarrow X$ is the generator of a $C_{0}$-semigroup of bounded operators $T(t)_{t>0}$ on a Banach space $(X,\|\cdot\|), x_{0} \in X$, $0=t_{0}=s_{0}<t_{1}<s_{1}<t_{2}<s_{2}<\ldots<t_{m} \leq s_{m}<t_{m+1}=T$ are fixed numbers, $g_{i} \in C(J \times X ; X), h, f,, k$ are given functions, $F, B, q: C(J ; X) \rightarrow C(J ; X)$ are given by $B x(t)=\int_{0}^{t} B(t, s) x(s) d s, F x(t)=\int_{0}^{t} F(t, s) x(s) d s$ and $\{F(t, s) ; t, s \in J\}$ $\{B(t, s) ; t, s \in J\}$ are a set of bounded linear operators on $X$ such that:

$F(t,). x \in C([0, t] ; X), \quad F(., s) \in C([s, T] ; X)$ for all $t, s \in J, B(t,). x \in C([0, t] ; X)$, $B(., s) \in C([s, T] ; X)$ for all $t, s \in J$ and $F^{*}=\sup _{t \in J} \int_{0}^{t}\|F(t, s)\|_{L(X)} d s$,

$B^{*}=\sup _{t \in J} \int_{0}^{t}\|B(t, s)\|_{L(X)} d s, \quad q^{*}=\sup _{s \in J} \int_{0}^{s}\|q(s-t)\| d t$.

In our work we will need the continuity of $x(t)$ at both points $t=s_{i}$ and $t=t_{i}$.

The rest of the paper is organized as follows. In section 2 we present the notations, definitions and preliminaries results needed in the following sections. In section 3, a suitable concept of mild solutions for our problems is introduced. Section 4 is concerned with the existence results of our problems.

\section{Preliminaries}

Let us set $J=[0, T], J_{0}=\left[0, t_{1}\right], J_{1}=\left(t_{1}, t_{2}\right], \ldots, J_{m-1}=\left(t_{m-1}, t_{m}\right], J_{m}=\left(t_{m}, t_{m+1}\right]$ and introduce the space $P C(J, X):=\left\{u: J \rightarrow X \mid u \in C\left(J_{k}, X\right), k=0,1,2, \ldots, m\right.$, and there exists $u\left(t_{k}^{+}\right)$and $u\left(t_{k}^{-}\right), k=1,2, \ldots, m$, with $\left.u\left(t_{k}^{-}\right)=u\left(t_{k}^{+}\right)\right\}$. It is clear that $P C(J, X)$ is a Banach space with the norm $\|u\|_{P C}=\sup \{\|u(t)\|: t \in J\}$.

Let $Y$ be a separable reflexive Banach space where controls $u$ takes values, and $P_{f}(Y)$ is a class of nonempty closed and convex subsets of $Y$. We suppose that the multivalued map $w:[0, a] \longrightarrow P_{f}(Y)$ is measurable, $w(.) \subset E$, where $E$ is bounded set of $Y$, and the admissible control set

$U_{a d}=\left\{c \in L^{p}(E): c(t) \in w(t), a . e\right\}, \quad p>\frac{1}{\tau},(\tau \in(0, \alpha))$, for more detail about admissible control set, we refer the readers to [13].

Lemma 2.1. (Theorem 2.1 in [11]) Suppose $W \subseteq P C(J, X)$. If the following conditions are satisfied:

(1) $W$ is uniformly bounded subset of $P C(J, X)$;

(2) $W$ is equicontinuous in $\left(t_{i}, t_{i+1}\right), i=0,1,2, . ., m$, where $t_{0}=0, t_{m+1}=T$; 
(3) $W(t)=\left\{u(t): u \in W, t \in J \backslash\left\{t_{1}, t_{2}, \ldots, t_{m}\right\}\right\}, W\left(t_{i}^{+}\right)=\left\{u\left(t_{i}^{+}\right): u \in W\right\}$ and $W\left(t_{i}^{-}\right)=\left\{u\left(t_{i}^{-}\right): u \in W\right\}, i=1,2, . ., m$, are relatively compact subsets of $X$.

Then $W$ is a relatively compact subset of $P C(J, X)$.

Let us recall the following well-known definitions.

Definition 2.2. ([6]) The Riemann-Liouville fractional integral of order q with lower limit zero for a function $\mathrm{f}$ is defined as

$I^{q} f(t)=\frac{1}{\Gamma(q)} \int_{0}^{t}(t-s)^{q-1} f(s) d s, q>0$,

provided the integral exists, where $\Gamma($.$) is the gamma function.$

Definition 2.3. ([6]) The Riemann-Liouville derivative of order $q$ with the lower limit zero for a function $f:[0, \infty) \rightarrow \mathbb{R}$ can be written as

${ }^{L} D^{q} f(t)=\frac{1}{\Gamma(n-q)} \frac{d^{n}}{d t^{n}} \int_{0}^{t}(t-s)^{n-q-1} f(s) d s, n-1<q<n, t>0$.

Definition 2.4. ([6]) The Caputo derivative of order q for a function $f:[0, \infty) \longrightarrow \mathbb{R}$ can be written as

${ }^{c} D^{q} f(t)={ }^{L} D^{q}\left(f(t)-\sum_{k=0}^{k} \frac{t^{k}}{k !} f^{k}(0)\right), n-1<q<n, t>0$.

Definition 2.5. ([12]-[14]) A function $x \in C(J, X)$ is said to be a mild solution of the following problem:

$$
\left\{\begin{array}{l}
{ }^{c} D^{\alpha} x(t)=A x(t)+y(t), t \in(0, T] \\
x(0)=x_{0}
\end{array}\right.
$$

If it satisfies the integral equation

$x(t)=P_{\alpha}(t) x_{0}+\int_{0}^{t}(t-s)^{\alpha-1} Q_{\alpha}(t-s) y(s) d s$.

Here

$$
\begin{aligned}
& P_{\alpha}(t)=\int_{0}^{\infty} \xi_{\alpha}(\theta) T\left(t^{\alpha} \theta\right) d \theta, Q_{\alpha}(t)=\alpha \int_{0}^{\infty} \theta \xi_{\alpha}(\theta) T\left(t^{\alpha} \theta\right) d \theta, \\
& \xi_{\alpha}(\theta)=\frac{1}{\alpha} \theta^{-1-\frac{1}{\alpha}} \bar{\omega}_{\alpha}\left(\theta^{-\frac{1}{\alpha}}\right) \geq 0, \\
& \bar{\omega}_{\alpha}(\theta)=\frac{1}{\pi} \sum_{n=1}^{\infty}(-1)^{n-1} \theta^{-n \alpha-1} \frac{\Gamma(n \alpha+1)}{n !} \sin (n \pi \alpha), \theta \in(0, \infty), \\
& \text { and } \xi_{\alpha}(\theta) \geq 0, \theta \in(0, \infty), \int_{0}^{\infty} \xi_{\alpha}(\theta) d \theta=1 .
\end{aligned}
$$

It is easy to verify that

$$
\int_{0}^{\infty} \theta \xi_{\alpha}(\theta) d \theta=\frac{1}{\Gamma(1+\alpha)} .
$$

We make the following assumption on $A$ in the whole paper.

$\mathrm{H}(1)$ : The operator $A$ generates a strongly continuous semigroup $\{T(t): t \geq 0\}$ in $\mathrm{X}$, and there is a constant $M_{A} \geq 1$ such that $\sup _{t \in[0, \infty)}\|T(t)\|_{L(X)} \leq M_{A}$. For any $t>0, T(t)$ is compact. 
Lemma 2.6. ([12]-[14]) Let $H(A)$ hold, then the operator $P_{\alpha}$ and $Q_{\alpha}$ have the following properties:

(1) For any fixed $t \geq 0, P_{\alpha}(t)$ and $Q_{\alpha}(t)$ are linear and bounded operators, and for any $x \in X$,

$\left\|P_{\alpha}(t) x\right\| \leq M_{A}\|x\|,\left\|Q_{\alpha}(t) x\right\| \leq \frac{\alpha M_{A}}{\Gamma(1+\alpha)}\|x\|$,

(2) $\left\{P_{\alpha}(t), t \geq 0\right\}$ and $\left\{Q_{\alpha}(t), t \geq 0\right\}$ are strongly continuous,

(3) for every $t>0, P_{\alpha}(t)$ and $Q_{\alpha}(t)$ are compact operators.

We recall a fixed point theorem which will be needed in the sequel.

Theorem 2.7. (Krasnoselskii fixed point theorem) Let $M$ be a closed, convex, and non-empty subset of a Banach space $X$. Let A,B be the operators such that:

(a) $A x+B y \in M$ for all $x, y \in M$,

(b) A is compact and continuous,

(c) $B$ is a contraction.

Then there exists a $x \in M$ such that $x=A x+B x$.

\section{The CONSTRUCTION OF MILD SOLUtions}

Let $x \in P C(J, X)$. We first consider the following fractional impulsive problem:

$$
\left\{\begin{array}{l}
{ }^{c} D^{\alpha} x(t)=A x(t)+f(t, x(t), F x(t), B x(t))+\int_{0}^{t} q(t-s) k(s, x(s)) d s+C(t) u(t), t \in\left(s_{i}, t_{i+1}\right], \\
i=0,1,2, \ldots, m, u \in U_{a d} \\
{ }^{c} D^{\beta} x(t)=g_{i}(t, x(t)), t \in\left(t_{i}, s_{i}\right], i=1,2, \ldots, m, \\
x(0)=x_{0}+h(x) .
\end{array}\right.
$$

From the property of the Caputo derivative, a general solution can be written as

$$
x(t)=\left\{\begin{array}{l}
x_{0}+h(x)+\frac{1}{\Gamma(\alpha} \int_{0}^{t}(t-s)^{\alpha-1}\left[A x(s)+f(s, x(s), F x(s), B x(s))+\int_{0}^{s} q(s-\tau) k(\tau, x(\tau)) d \tau+\right. \\
C(s) u(s)] d s, t \in\left(0, t_{1}\right], \\
d_{1 x}+\frac{1}{\Gamma(\beta)} \int_{0}^{t}(t-s)^{\beta-1} g_{1}(s, x(s)) d s, t \in\left(t_{1}, s_{1}\right], \\
K_{1 x}+\frac{1}{\Gamma(\alpha)} \int_{0}^{t}(t-s)^{\alpha-1}\left[A x(s)+f(s, x(s), F x(s), B x(s))+\int_{0}^{s} q(s-\tau) k(\tau, x(\tau)) d \tau+\right. \\
C(s) u(s)] d s, t \in\left(s_{1}, t_{2}\right], \\
\cdot \\
d_{i x}+\frac{1}{\Gamma(\beta)} \int_{0}^{t}(t-s)^{\beta-1} g_{i}(s, x(s)) d s, t \in\left(t_{i}, s_{i}\right], \\
K_{i x}+\frac{1}{\Gamma(\alpha)} \int_{0}^{t}(t-s)^{\alpha-1}\left[A x(s)+f(s, x(s), F x(s), B x(s))+\int_{0}^{s} q(s-\tau) k(\tau, x(\tau)) d \tau+\right. \\
C(s) u(s)] d s, t \in\left(s_{i}, t_{i+1}\right],
\end{array}\right.
$$


where $d_{i x}$ and $K_{i x}, i=1,2, \ldots, m$, are elements of X.

Using [3] pages 5 and 6 we obtain:

$x(t)=\left\{\begin{array}{l}d_{i x}+\frac{1}{\Gamma(\beta)} \int_{0}^{t}(t-s)^{\beta-1} g_{i}(s, x(s)) d s, t \in\left(t_{i}, s_{i}\right], 1 \leq i \leq m, \\ P_{\alpha}\left(t-s_{i}\right) K_{i x}+\int_{0}^{t}(t-s)^{\alpha-1} Q_{\alpha}(t-s)\left[f(s, x(s), F x(s), B x(s))+\int_{0}^{s} q(s-\tau) k(\tau, x(\tau)) d \tau+\right. \\ +C(s) u(s)] d s, t \in\left(s_{i}, t_{i+1}\right], 0 \leq i \leq m, \\ K_{0 x}=x(0) .\end{array}\right.$

And using the fact that $\mathrm{x}$ is continuous at the points $t_{i}$, we get :

$$
\begin{aligned}
x\left(t_{i}\right)= & P_{\alpha}\left(t_{i}-s_{i-1}\right) K_{(i-1) x}+\int_{0}^{t_{i}}\left(t_{i}-s\right)^{\alpha-1} Q_{\alpha}\left(t_{i}-s\right)[f(s, x(s), F x(s), B x(s))+ \\
& \left.+\int_{0}^{s} q(s-\tau) k(\tau, x(\tau)) d \tau+C(s) u(s)\right] d s \\
= & d_{i x}+\frac{1}{\Gamma(\beta)} \int_{0}^{t_{i}}\left(t_{i}-s\right)^{\beta-1} g_{i}(s, x(s)) d s .
\end{aligned}
$$

Which implies that:

$$
\begin{aligned}
d_{i x}= & P_{\alpha}\left(t_{i}-s_{i-1}\right) K_{(i-1) x}+\int_{0}^{t_{i}}\left(t_{i}-s\right)^{\alpha-1} Q_{\alpha}\left(t_{i}-s\right)[f(s, x(s), F x(s), B x(s))+ \\
& \left.+\int_{0}^{s} q(s-\tau) k(\tau, x(\tau)) d \tau+C(s) u(s)\right] d s-\frac{1}{\Gamma(\beta)} \int_{0}^{t_{i}}\left(t_{i}-s\right)^{\beta-1} g_{i}(s, x(s)) d s .
\end{aligned}
$$

Using the fact that $\mathrm{x}$ is continuous at the points $s_{i}$, we get :

$$
\begin{aligned}
x\left(s_{i}\right) & =d_{i x}+\frac{1}{\Gamma(\beta)} \int_{0}^{s_{i}}\left(s_{i}-s\right)^{\beta-1} g_{i}(s, x(s)) d s \\
& =K_{i x}+\int_{0}^{s_{i}}\left(s_{i}-s\right)^{\alpha-1} Q_{\alpha}\left(s_{i}-s\right)\left[f(s, x(s), F x(s), B x(s))+\int_{0}^{s} q(s-\tau) k(\tau, x(\tau)) d \tau+C(s) u(s)\right] d s
\end{aligned}
$$

Which implies that:

$$
\begin{aligned}
K_{i x}= & d_{i x}+\frac{1}{\Gamma(\beta)} \int_{0}^{s_{i}}\left(s_{i}-s\right)^{\beta-1} g_{i}(s, x(s)) d s- \\
& -\int_{0}^{s_{i}}\left(s_{i}-s\right)^{\alpha-1} Q_{\alpha}\left(s_{i}-s\right)\left[f(s, x(s), F x(s), B x(s))+\int_{0}^{s} q(s-\tau) k(\tau, x(\tau)) d \tau+C(s) u(s)\right] d s
\end{aligned}
$$

Therefore, a mild solution of problem (1.2) is given by

$$
x(t)=\left\{\begin{array}{l}
P_{\alpha}(t)\left(x_{0}+h(x)\right)+\int_{0}^{t}(t-s)^{\alpha-1} Q_{\alpha}(t-s)\left[f(s, x(s), F x(s), B x(s))+\int_{0}^{s} q(s-\tau) k(\tau, x(\tau)) d \tau+\right. \\
C(s) u(s)] d s, t \in\left(0, t_{1}\right], \\
d_{1 x}+\frac{1}{\Gamma(\beta)} \int_{0}^{t}(t-s)^{\beta-1} g_{1}(s, x(s)) d s, t \in\left(t_{1}, s_{1}\right], \\
P_{\alpha}\left(t-s_{1}\right) K_{1 x}+\int_{0}^{t}(t-s)^{\alpha-1} Q_{\alpha}(t-s)\left[f(s, x(s), F x(s), B x(s))+\int_{0}^{s} q(s-\tau) k(\tau, x(\tau)) d \tau+\right. \\
C(s) u(s)] d s, t \in\left(s_{1}, t_{2}\right], \\
\cdot \\
\cdot \\
d_{i x}+\frac{1}{\Gamma(\beta)} \int_{0}^{t}(t-s)^{\beta-1} g_{i}(s, x(s)) d s, t \in\left(t_{i}, s_{i}\right], 1 \leq i \leq m, \\
P_{\alpha}\left(t-s_{i}\right) K_{i x}+\int_{0}^{t}(t-s)^{\alpha-1} Q_{\alpha}(t-s)\left[f(s, x(s), F x(s), B x(s))+\int_{0}^{s} q(s-\tau) k(\tau, x(\tau)) d \tau+\right. \\
C(s) u(s)] d s, \\
t \in\left(s_{i}, t_{i+1}\right], 1 \leq i \leq m,
\end{array}\right.
$$

where

$$
\begin{aligned}
K_{0 x}=x_{0} & +h(x) \\
d_{i x}= & P_{\alpha}\left(t_{i}-s_{i-1}\right) K_{(i-1) x}+\int_{0}^{t_{i}}\left(t_{i}-s\right)^{\alpha-1} Q_{\alpha}\left(t_{i}-s\right)\left[f(s, x(s), F x(s), B x(s))+\int_{0}^{s} q(s-\tau) k(\tau, x(\tau)) d \tau+\right. \\
& +C(s) u(s)] d s-\frac{1}{\Gamma(\beta)} \int_{0}^{t_{i}}\left(t_{i}-s\right)^{\beta-1} g_{i}(s, x(s)) d s \\
K_{i x}= & d_{i x}-\int_{0}^{s_{i}}\left(s_{i}-s\right)^{\alpha-1} Q_{\alpha}\left(s_{i}-s\right)\left[f(s, x(s), F x(s), B x(s))+\int_{0}^{s} q(s-\tau) k(\tau, x(\tau)) d \tau+C(s) u(s)\right] d s+ \\
& +\frac{1}{\Gamma(\beta)} \int_{0}^{s_{i}}\left(s_{i}-s\right)^{\beta-1} g_{i}(s, x(s)) d s
\end{aligned}
$$


Definition 3.1. A function $x \in P C(J ; X)$ is said to be a mild solution of problem (1.2) if it satisfies the following relation:

$$
x(t)=\left\{\begin{array}{l}
P_{\alpha}(t) K_{0 x}+\int_{0}^{t}(t-s)^{\alpha-1} Q_{\alpha}(t-s)\left[f(s, x(s), F x(s), B x(s))+\int_{0}^{s} q(s-\tau) k(\tau, x(\tau)) d \tau+\right. \\
C(s) u(s)] d s, t \in\left(0, t_{1}\right], \\
d_{i x}+\frac{1}{\Gamma(\beta)} \int_{0}^{t}(t-s)^{\beta-1} g_{i}(s, x(s)) d s, t \in\left(t_{i}, s_{i}\right], 1 \leq i \leq m, \\
P_{\alpha}\left(t-s_{i}\right) K_{i x}+\int_{0}^{t}(t-s)^{\alpha-1} Q_{\alpha}(t-s)\left[f(s, x(s), F x(s), B x(s))+\int_{0}^{s} q(s-\tau) k(\tau, x(\tau)) d \tau+\right. \\
C(s) u(s)] d s, t \in\left(s_{i}, t_{i+1}\right], 1 \leq i \leq m .
\end{array}\right.
$$

Where

$$
\begin{aligned}
K_{0 x}= & x_{0}+h(x) \\
d_{i x}= & P_{\alpha}\left(t_{i}-s_{i-1}\right) K_{(i-1) x}+\int_{0}^{t_{i}}\left(t_{i}-s\right)^{\alpha-1} Q_{\alpha}\left(t_{i}-s\right)\left[f(s, x(s), F x(s), B x(s))+\int_{0}^{s} q(s-\tau) k(\tau, x(\tau)) d \tau+\right. \\
& C(s) u(s)] d s-\frac{1}{\Gamma(\beta)} \int_{0}^{t_{i}}\left(t_{i}-s\right)^{\beta-1} g_{i}(s, x(s)) d s \\
K_{i x}= & d_{i x}-\int_{0}^{s_{i}}\left(s_{i}-s\right)^{\alpha-1} Q_{\alpha}\left(s_{i}-s\right)\left[f(s, x(s), F x(s), B x(s))+\int_{0}^{s} q(s-\tau) k(\tau, x(\tau)) d \tau+C(s) u(s)\right] d s+ \\
& \frac{1}{\Gamma(\beta)} \int_{0}^{s_{i}}\left(s_{i}-s\right)^{\beta-1} g_{i}(s, x(s)) d s .
\end{aligned}
$$

Here

$$
\begin{aligned}
& P_{\alpha}(t)=\int_{0}^{\infty} \xi_{\alpha}(\theta) T\left(t^{\alpha} \theta\right) d \theta, Q_{\alpha}(t)=\alpha \int_{0}^{\infty} \theta \xi_{\alpha}(\theta) T\left(t^{\alpha} \theta\right) d \theta, \\
& \xi_{\alpha}(\theta)=\frac{1}{\alpha} \theta^{-1-\frac{1}{\alpha}} \bar{\omega}_{\alpha}\left(\theta^{-\frac{1}{\alpha}}\right) \geq 0, \\
& \overline{\omega_{\alpha}}(\theta)=\frac{1}{\pi} \sum_{n=1}^{\infty}(-1)^{n-1} \theta^{-n \alpha-1} \frac{\Gamma(n \alpha+1)}{n !} \sin (n \pi \alpha), \theta \in(0, \infty), \\
& \text { and } \xi_{\alpha}(\theta) \geq 0, \theta \in(0, \infty), \int_{0}^{\infty} \xi_{\alpha}(\theta) d \theta=1 .
\end{aligned}
$$

It is easy to verify that

$$
\int_{0}^{\infty} \theta \xi_{\alpha}(\theta) d \theta=\frac{1}{\Gamma(1+\alpha)} .
$$

We make the following assumption on $A$ in the whole paper.

$\mathrm{H}(1)$ : The operator $A$ generates a strongly continuous semigroup $\{T(t): t \geq 0\}$ in $\mathrm{X}$, and there is a constant $M_{A} \geq 1$ such that $\sup _{t \in[0, \infty)}\|T(t)\|_{L(X)} \leq M_{A}$. For any $t>0, T(t)$ is compact.

Lemma 3.2. ([12]-[14]) Let $H(A)$ hold, then the operator $P_{\alpha}$ and $Q_{\alpha}$ have the following properties:

(1) For any fixed $t \geq 0, P_{\alpha}(t)$ and $Q_{\alpha}(t)$ are linear and bounded operators, and for any $x \in X$, $\left\|P_{\alpha}(t) x\right\| \leq M_{A}\|x\|,\left\|Q_{\alpha}(t) x\right\| \leq \frac{\alpha M_{A}}{\Gamma(1+\alpha)}\|x\|$,

(2) $\left\{P_{\alpha}(t), t \geq 0\right\}$ and $\left\{Q_{\alpha}(t), t \geq 0\right\}$ are strongly continuous,

(3) for every $t>0, P_{\alpha}(t)$ and $Q_{\alpha}(t)$ are compact operators.

We recall a fixed point theorem which will be needed in the sequel. 
Theorem 3.3. (Krasnoselskii fixed point theorem) Let $M$ be a closed, convex, and non-empty subset of a Banach space X. Let A,B be the operators such that:

(a) $A x+B y \in M$ for all $x, y \in M$,

(b) $A$ is compact and continuous,

(c) $B$ is a contraction.

Then there exists a $x \in M$ such that $x=A x+B x$.

\section{Existence And Uniqueness of Mild Solution}

This section deals with the existence results for the problem (1.2).

From Definition (3.1), we define an operator $P: P C(J, X) \rightarrow P C(J, X)$ as

$P x(t)=\left\{\begin{array}{l}P_{\alpha}(t)\left(x_{0}+h(x)\right)+\int_{0}^{t}(t-s)^{\alpha-1} Q_{\alpha}(t-s)\left[f(s, x(s), F x(s), B x(s))+\int_{0}^{s} q(s-\tau) k(\tau, x(\tau)) d \tau+\right. \\ C(s) u(s)] d s, t \in\left[0, t_{1}\right], \\ d_{i x}+\frac{1}{\Gamma(\beta)} \int_{0}^{t}(t-s)^{\beta-1} g_{i}(s, x(s)) d s, t \in\left(t_{i}, s_{i}\right], \\ P_{\alpha}\left(t-s_{i}\right) K_{i x}+\int_{0}^{t}(t-s)^{\alpha-1} Q_{\alpha}(t-s)\left[f(s, x(s), F x(s), B x(s))+\int_{0}^{s} q(s-\tau) k(\tau, x(\tau)) d \tau+\right. \\ C(s) u(s)] d s, t \in\left(s_{i}, t_{i+1}\right] .\end{array}\right.$

To prove our first existence result we introduce the following assumptions.

$\left(H_{2}\right) C:[0, T] \longrightarrow L(Y, X)$ is essentially bounded, ie $C \in L^{\infty}([0, T], L(Y, X))$,

$\left(H_{3}\right)$ The functions $f \in C(J \times X \times X \times X ; X)$, and $k \in C(J \times X ; X)$,

$\left(H_{4}\right)$ there exists $C_{f}, L_{f}, M_{f}, L_{k}>0$ such that

- $\left\|f\left(t, x_{1}, y_{1}, z_{1}\right)-f\left(t, x_{2}, y_{2}, z_{2}\right)\right\| \leq L_{f}\left\|x_{1}-x_{2}\right\|+C_{f}\left\|y_{1}-y_{2}\right\|+M_{f} \|$ $z_{1}-z_{2} \|$, for all $x_{1}, x_{2}, y_{1}, y_{2}, z_{1}, z_{2} \in X$, and $t \in J$,

- $\left\|k\left(t, x_{1}\right)-k\left(t, x_{2}\right)\right\| \leq L_{k}\left\|x_{1}-x_{2}\right\|$, for all $x_{1}, x_{2} \in X$, and $t \in J$,

$\left(H_{5}\right)$ There exists a constant $D, D^{\prime}>0$ such that

- $\|f(t, x, y, z)\| \leq D\left(1+\|x\|^{\mu}+\|y\|^{\nu}+\|z\|^{\phi}\right)$ for every $t \in J$ and $x, y, z \in X, \mu, \nu, \phi \in[0,1]$,

- $\|k(t, x)\| \leq D^{\prime}\left(1+\|x\|^{\mu}\right)$ for every $t \in J$ and $x \in X, \mu \in[0,1]$,

$\left(H_{6}\right)$

- For $i=1,2, \ldots, m, g_{i} \in C(J \times X ; X)$, and there exists $L_{g}>0$ such that $\left\|g_{i}(t, x)-g_{i}(t, y)\right\| \leq L_{g}\|x-y\|$, for all $x, y \in X$,

- There exists a function $t \longrightarrow \varphi_{i}(t), i=1,2, \ldots, m$ such that $\left\|g_{i}(t, x(t))\right\| \leq \varphi_{i}(t)$ for all $t \in J, x \in X$ and $L_{g_{i}}=\sup _{t \in J} \varphi_{i}(t)$ and $L_{g}^{\prime}=\max _{1 \leq i \leq m} L_{g_{i}}$

- $h: P C(J ; X) \rightarrow X$ and there exists a constant $L_{h}>0$ and $\varphi_{h} \in C\left([0, \infty) ; \mathbb{R}^{+}\right)$ such that for $x, y \in P C(J ; X),\|h(x)-h(y)\| \leq L_{h}\|x-y\|_{P C}$;

$\|h(x)\| \leq \varphi_{h}(t)$ and $L_{h}^{\prime}=\sup _{t \in J} \varphi_{h}(t)$.

Theorem 4.1. Assume that $\left(H_{1}\right)-\left(H_{4}\right),\left(H_{6}\right)$ are satisfied and

$\left[M^{m+1} L_{h}+\frac{M T^{\alpha}+M^{2}\left(t_{m}^{\alpha}+s_{m}^{\alpha}\right)+. .+M^{m+1}\left(t_{1}^{\alpha}+s_{1}^{\alpha}\right)}{\Gamma(\alpha+1)}\left(L_{f}+q^{*} L_{k}+M_{f} B^{*}+C_{f} F^{*}\right)+\frac{M L_{g}\left(t_{m}^{\beta}+s_{m}^{\beta}\right)+. .+M^{m} L_{g}\left(t_{1}^{\beta}+s_{1}^{\beta}\right)}{\Gamma(\beta+1)}\right]<1$.

Then there exists a unique mild solution of problem (1.2). 
Proof. From the assumptions it is easy to show that the operator $P$ is well defined on $P C(J, X)$.

Let $x, y \in P C(J, X)$.

Case 1. For $t \in\left[0, t_{1}\right]$, we have

$$
\begin{aligned}
\|(P x)(t)-(P y)(t)\| \leq & \left\|P_{\alpha}(t)\left(x_{0}+h(x)-x_{0}-h(y)\right)\right\|+ \\
& +\| \int_{0}^{t}(t-s)^{\alpha-1} Q_{\alpha}(t-s)\left[f(s, x(s), F x(s), B x(s))+\int_{0}^{s} q(s-\tau) k(\tau, x(\tau)) d \tau+\right. \\
& \left.C(s) u(s)-f(s, y(s), F y(s), B y(s))-\int_{0}^{s} q(s-\tau) k(\tau, y(\tau)) d \tau-C(s) u(s)\right] d s \| \\
\leq & M_{A}\|h(x)-h(y)\|+\frac{\alpha M_{A}}{\Gamma(\alpha+1)} \int_{0}^{t}(t-s)^{\alpha-1} \| f(s, x(s), F x(s), B x(s))- \\
& f(s, y(s), F y(s), B y(s))+\int_{0}^{s} q(s-\tau) k(\tau, x(\tau)) d \tau-\int_{0}^{s} q(s-\tau) k(\tau, y(\tau)) d \tau \| d s \\
\leq & M_{A} L_{h}\|x-y\|_{P C}+\frac{\alpha M_{A}}{\Gamma(\alpha+1)} \int_{0}^{t}(t-s)^{\alpha-1}\left(L_{f}\|x(s)-y(s)\|+\right. \\
& \left.C_{f}\|F x(s)-F y(s)\|+M_{f}\|B x(s)-B y(s)\|+q^{*} L_{k}\|x(s)-y(s)\|\right) d s \\
\leq & M_{A} L_{h}\|x-y\|_{P C}+\frac{M_{A} t_{1}^{\alpha}}{\Gamma(\alpha+1)}\left(L_{f}+q^{*} L_{k}+M_{f} B^{*}+C_{f} F^{*}\right)\|x-y\|_{P C} \\
\leq & {\left[M_{A} L_{h}+\frac{M_{A} t_{1}^{\alpha}}{\Gamma(\alpha+1)}\left(L_{f}+q^{*} L_{k}+M_{f} B^{*}+C_{f} F^{*}\right)\right]\|x-y\|_{P C} . }
\end{aligned}
$$

Case 2. For $t \in\left(t_{i}, s_{i}\right] \cup\left(s_{i}, t_{i+1}\right]$.

We prove that for $t \in\left(t_{i}, s_{i}\right], i=1,2, \ldots, m$ :

$$
\begin{aligned}
\|(P x)(t)-(P y)(t)\| \leq & {\left[M_{A}^{i} L_{h}+\frac{M_{A} t_{i}^{\alpha}+M_{A}^{2}\left(t_{i-1}^{\alpha}+s_{i-1}^{\alpha}\right)+M_{A}^{3}\left(t_{i-2}^{\alpha}+s_{i-2}^{\alpha}\right)+\ldots+M_{A}^{i}\left(t_{1}^{\alpha}+s_{1}^{\alpha}\right)}{\Gamma(\alpha+1)} \times\right.} \\
& \left(L_{f}+q^{*} L_{k}+M_{f} B^{*}+C_{f} F^{*}\right)+ \\
& \left.\frac{L_{g}\left(t_{i}^{\beta}+s_{i}^{\beta}\right)+M_{A} L_{g}\left(t_{i-1}^{\beta}+s_{i-1}^{\beta}\right)+\ldots+M_{A}^{i-2} L_{g}\left(t_{2}^{\beta}+s_{2}^{\beta}\right)+M_{A}^{i-1} L_{g}\left(t_{1}^{\beta}+s_{1}^{\beta}\right)}{\Gamma(\beta+1)}\right] \times \\
& \|x-y\|_{P C},
\end{aligned}
$$

and for $t \in\left[s_{i}, t_{i+1}\right], i=1,2, \ldots, m$,

$$
\begin{aligned}
\|(P x)(t)-(P y)(t)\| \leq & {\left[M_{A}^{i+1} L_{h}+\frac{M_{A} t_{i+1}^{\alpha}+M_{A}^{2}\left(t_{i}^{\alpha}+s_{i}^{\alpha}\right)+\ldots+M_{A}^{i}\left(t_{2}^{\alpha}+s_{2}^{\alpha}\right)+M_{A}^{i+1}\left(t_{1}^{\alpha}+s_{1}^{\alpha}\right)}{\Gamma(\alpha+1)} \times\right.} \\
& \left(L_{f}+q^{*} L_{k}+M_{f} B^{*}+C_{f} F^{*}\right)+ \\
& \left.\frac{M_{A} L_{g}\left(t_{i}^{\beta}+s_{i}^{\beta}\right)+\ldots+M_{A}^{i-1} L_{g}\left(t_{2}^{\beta}+s_{2}^{\beta}\right)+M_{A}^{i} L_{g}\left(t_{1}^{\beta}+s_{1}^{\beta}\right)}{\Gamma(\beta+1)}\right]\|x-y\|_{P C} .
\end{aligned}
$$

For $t \in\left(t_{1}, s_{1}\right]$

$$
\begin{aligned}
& \|(P x)(t)-(P y)(t)\| \leq\left\|d_{1} x+\frac{1}{\Gamma(\beta)} \int_{0}^{t}(t-s)^{\beta-1} g_{1}(s, x(s)) d s-d_{1} y-\frac{1}{\Gamma(\beta)} \int_{0}^{t}(t-s)^{\beta-1} g_{1}(s, y(s)) d s\right\| \\
& \leq\left\|d_{1} x-d_{1} y\right\|+\frac{1}{\Gamma(\beta)} \int_{0}^{t}(t-s)^{\beta-1}\left\|g_{1}(s, x(s))-g_{1}(s, y(s))\right\| d s \\
& \leq\left\|P_{\alpha}\left(t_{1}\right)(h(x)-h(y))\right\|+\| \int_{0}^{t_{1}}\left(t_{1}-s\right)^{\alpha-1} Q_{\alpha}\left(t_{1}-s\right)[f(s, x(s), F x(s), B x(s))- \\
& \left.-f(s, y(s), F y(s), B y(s))+\int_{0}^{s} q(s-\tau) k(\tau, x(\tau)) d \tau-\int_{0}^{s} q(s-\tau) k(\tau, y(\tau)) d \tau\right] d s \| \\
& +\left\|\frac{1}{\Gamma(\beta)} \int_{0}^{t_{1}}(t-s)^{\beta-1}\left(g_{1}(s, x(s))-g_{1}(s, y(s))\right) d s\right\|+ \\
& +\left\|\frac{1}{\Gamma(\beta)} \int_{0}^{t}(t-s)^{\beta-1}\left(g_{1}(s, x(s))-g_{1}(s, y(s))\right) d s\right\| \\
& \leq M_{A} L_{h}\|x-y\|_{P C}+\frac{M_{A} t_{1}^{\alpha}}{\Gamma(\alpha+1)}\left(L_{f}+q^{*} L_{k}+M_{f} B^{*}+C_{f} F^{*}\right)\|x-y\|_{P C}+ \\
& \frac{\left(s_{1}^{\beta}+t_{1}^{\beta}\right)}{\Gamma(\beta+1)} L_{g}\|x-y\|_{P C} \\
& \leq\left[M_{A} L_{h}+\frac{M_{A} t_{1}^{\alpha}}{\Gamma(\alpha+1)}\left(L_{f}+q^{*} L_{k}+M_{f} B^{*}+C_{f} F^{*}\right)+\frac{\left(s_{1}^{\beta}+t_{1}^{\beta}\right)}{\Gamma(\beta+1)} L_{g}\right]\|x-y\|_{P C} .
\end{aligned}
$$


For $t \in\left(s_{1}, t_{2}\right]$

$$
\begin{aligned}
\|P x(t)-P y(t)\| \leq & \left\|P_{\alpha}\left(t-s_{1}\right) K_{1 x}-P_{\alpha}\left(t-s_{1}\right) K_{1 y}\right\| \\
& +\| \int_{0}^{t}(t-s)^{\alpha-1} Q_{\alpha}(t-s)[\| f(s, x(s), F x(s), B x(s))-f(s, y(s), F y(s), B y(s)) \\
& \left.+\int_{0}^{s} q(s-\tau) k(\tau, x(\tau)) d \tau-\int_{0}^{s} q(s-\tau) k(\tau, y(\tau)) d \tau\right] d s \| \\
\leq & M_{A}\left\|d_{1 x}-d_{1 y}\right\|+M_{A} \| \int_{0}^{s_{1}}\left(s_{1}-s\right)^{\alpha-1} Q_{\alpha}\left(s_{1}-s\right)[f(s, x(s), F x(s), B x(s))- \\
& \left.f(s, y(s), F y(s), B y(s))+\int_{0}^{s} q(s-\tau) k(\tau, x(\tau)) d \tau-\int_{0}^{s} q(s-\tau) k(\tau, y(\tau)) d \tau\right] d s \|+ \\
& +M_{A}\left\|\frac{1}{\Gamma(\beta)} \int_{0}^{s_{1}}\left(s_{1}-s\right)^{\beta-1}\left[g_{1}(s, x(s))-g_{1}(s, y(s))\right] d s\right\| \\
& +\| \int_{0}^{t}(t-s)^{\alpha-1} Q_{\alpha}(t-s)[f(s, x(s), F x(s), B x(s))-f(s, y(s), F y(s), B y(s))+ \\
& \left.+\int_{0}^{s} q(s-\tau) k(\tau, x(\tau)) d \tau-\int_{0}^{s} q(s-\tau) k(\tau, y(\tau)) d \tau\right] d s \| \\
\leq & {\left[M_{A}^{2} L_{h}+\frac{\left.M_{A}^{2} t_{1}^{\alpha}+M_{A}^{2} s_{1}^{\alpha}+M_{A} t_{2}^{\alpha}\left(L_{f}+q^{*} L_{k}+M_{f} B^{*}+C_{f} F^{*}\right)+\frac{M_{A} L_{g}\left(s_{1}^{\beta}+t_{1}^{\beta}\right)}{\Gamma(\alpha+1)}\right]}{\Gamma(\beta+1)}\right] }
\end{aligned}
$$

We suppose that for $1 \leq j \leq i$ we have:

$$
\begin{aligned}
& \text { for } t \in\left(t_{j}, s_{j}\right] \\
& \qquad \begin{aligned}
\|P x(t)-P y(t)\| \leq & {\left[M_{A}^{j} L_{h}+\frac{M_{A} t_{j}^{\alpha}+M_{A}^{2}\left(t_{j-1}^{\alpha}+s_{j-1}^{\alpha}\right)+M_{A}^{3}\left(t_{j-2}^{\alpha}+s_{j-2}^{\alpha}\right)+. .+M_{A}^{j}\left(t_{1}^{\alpha}+s_{1}^{\alpha}\right)}{\Gamma(\alpha+1)} \times\right.} \\
& \left(L_{f}+q^{*} L_{k}+M_{f} B^{*}+C_{f} F^{*}\right) \\
& \left.\frac{L_{g}\left(t_{j}^{\beta}+s_{j}^{\beta}\right)+M_{A} L_{g}\left(t_{j-1}^{\beta}+s_{j-1}^{\beta}\right)+. .+M_{A}^{j-1} L_{g}\left(t_{1}^{\beta}+s_{1}^{\beta}\right)}{\Gamma(\beta+1)}\right]\|x-y\|_{P C},
\end{aligned}
\end{aligned}
$$

and for $t \in\left(s_{j}, t_{j+1}\right]$,

$$
\begin{aligned}
\|(P x)(t)-(P y)(t)\| \leq & M_{A}\left[M_{A}^{j} L_{h}+\frac{t_{j+1}^{\alpha}+M_{A}\left(t_{j}^{\alpha}+s_{j}^{\alpha}\right)+. .+M_{A}^{j}\left(t_{1}^{\alpha}+s_{1}^{\alpha}\right)}{\Gamma(\alpha+1)} \times\right. \\
& \left(L_{f}+q^{*} L_{k}+M_{f} B^{*}+C_{f} F^{*}\right)+ \\
& \left.\frac{L_{g}\left(t_{j}^{\beta}+s_{j}^{\beta}\right)+. .+M_{A}^{j-2} L_{g}\left(t_{2}^{\beta}+s_{2}^{\beta}\right)+M_{A}^{j-1} L_{g}\left(t_{1}^{\beta}+s_{1}^{\beta}\right)}{\Gamma(\beta+1)}\right]\|x-y\|_{P C} .
\end{aligned}
$$

We prove the relations for $j=i+1$.

For $t \in\left(t_{i+1}, s_{i+1}\right]$

$$
\begin{gathered}
\|P x(t)-P y(t)\| \leq \| d_{i+1} x+\frac{1}{\Gamma(\beta)} \int_{0}^{t}(t-s)^{\beta-1} g_{i+1}(s, x(s)) d s-d_{i+1} y- \\
\quad \frac{1}{\Gamma(\beta)} \int_{0}^{t}(t-s)^{\beta-1} g_{i+1}(s, y(s)) d s \| \\
\leq\left\|d_{i+1} x-d_{i+1} y\right\|+\frac{1}{\Gamma(\beta)} \int_{0}^{t}(t-s)^{\beta-1}\left\|g_{i+1}(s, x(s))-g_{i+1}(s, y(s))\right\| d s \\
. \quad\left\|P_{\alpha}\left(t_{i+1}-s_{i}\right)\left(K_{i x}-K_{i y}\right)\right\|+\| \int_{0}^{t_{i+1}}\left(t_{i+1}-s\right)^{\alpha-1} Q_{\alpha}\left(t_{i+1}-s\right) \times \\
\quad\left[f(s, x(s), F x(s), B x(s))-f_{0}^{s}(s, y(s), F y(s), B y(s))+\right. \\
\left.\quad \int_{0}^{s} q(s-\tau) k(\tau, x(\tau)) d \tau-\int_{0}^{s} q(s-\tau) k(\tau, y(\tau)) d \tau\right] d s \| \\
\quad+\| \frac{1}{\Gamma(\beta)} \int_{0}^{t_{i+1}}\left(t_{i+1}-s\right)^{\beta-1}\left(g_{i+1}(s, x(s))-g_{i+1}(s, y(s)) d s \|+\right. \\
\quad+\frac{1}{\Gamma(\beta)} \int_{0}^{t}(t-s)^{\beta-1}\left\|\left(g_{i+1}(s, x(s))-g_{i+1}(s, y(s))\right)\right\| d s \\
\leq \quad\left[M_{A}^{i+1} L_{h}+\frac{M_{A} t_{i+1}^{\alpha}+M_{A}^{2}\left(t_{i}^{\alpha}+s_{i}^{\alpha}\right)+\ldots+M_{A}^{i+1}\left(t_{1}^{\alpha}+s_{1}^{\alpha}\right)}{\Gamma(\alpha+1)} \times\right. \\
\quad\left(L_{f}+q^{*} L_{k}+M_{f} B^{*}+C_{f} F^{*}\right)+ \\
\left.\quad \frac{L_{g}\left(t_{i+1}^{\beta}+s_{i+1}^{\beta}\right)+M_{A}^{i} L_{g}\left(t_{1}^{\beta}+s_{1}^{\beta}\right)+\ldots+M_{A} L_{g}\left(t_{i}^{\beta}+s_{i}^{\beta}\right)}{\Gamma(\beta+1)}\right]\|x-y\|_{P C} . \\
t \in\left(s_{i+1}, t_{i+2}\right]
\end{gathered}
$$




$$
\begin{aligned}
\|P x(t)-P y(t)\| \leq & \left\|P_{\alpha}\left(t-s_{i+1}\right) K_{(i+1) x}-P_{\alpha}\left(t-s_{i+1}\right) K_{(i+1) y}\right\| \\
& +\| \int_{0}^{t}(t-s)^{\alpha-1} Q_{\alpha}(t-s)[f(s, x(s), F x(s), B x(s))-f(s, y(s), F y(s), B y(s)) \\
& \left.+\int_{0}^{s} q(s-\tau) k(\tau, x(\tau)) d \tau-\int_{0}^{s} q(s-\tau) k(\tau, y(\tau)) d \tau\right] d s \| \\
\leq & \leq M_{A}\left[\left\|d_{(i+1) x}-d_{(i+1) y}\right\|+\frac{s_{i+1}^{\alpha} M_{A}}{\Gamma(\alpha+1)}\left(L_{f}+q^{*} L_{k}+M_{f} B^{*}+C_{f} F^{*}\right)\|x-y\|_{P C}+\right. \\
& \left.+\frac{s_{i+1}^{\beta}}{\Gamma(\beta+1)} L_{g}\|x-y\|_{P C}\right]+\frac{t_{i+2}^{\alpha} M_{A}}{\Gamma(\alpha+1)}\left(L_{f}+q^{*} L_{k}+M_{f} B^{*}+C_{f} F^{*}\right)\|x-y\|_{P C} \\
\leq & {\left[M _ { A } \left[M_{A}^{i+1} L_{h}+\frac{t_{i+2}^{\alpha}+M_{A}^{i+1}\left(t_{1}^{\alpha}+s_{1}^{\alpha}\right)+\ldots+M_{A}^{2}\left(t_{i}^{\alpha}+s_{i}^{\alpha}\right)+M_{A}\left(t_{i+1}^{\alpha}+s_{i+1}^{\alpha}\right)}{\Gamma(\alpha+1)} \times\right.\right.} \\
& \left.\times\left(L_{f}+q^{*} L_{k}+M_{f} B^{*}+C_{f} F^{*}\right)\right]+ \\
& \left.+\frac{M_{A} L_{g}\left(s_{i+1}^{\beta}+t_{i+1}^{\beta}\right)+M_{A}^{2} L_{g}\left(s_{i}^{\beta}+t_{i}^{\beta}\right)+. .+M_{A}^{i+1} L_{g}\left(t_{1}^{\beta}+s_{1}^{\beta}\right)}{\Gamma(\beta+1)}\right]\|x-y\|_{P C} .
\end{aligned}
$$

Then it follows that $P$ is a contraction on the space $P C(J, X)$. Hence by the Banach contraction mapping principale, $P$ has a unique fixed point $x \in P C(J, X)$ which is the unique mild solution of problem (1.2) .

The next result is based on Krasnoselskii fixed point theorem.

Theorem 4.2. Assume that $\left(H_{1}\right)-\left(H_{3}\right)$, and $\left(H_{5}\right)-\left(H_{6}\right)$ hold. In addition, let's suppose that the following condition is verified:

$$
\begin{aligned}
& \max \left\{M_{A}^{m+1} L_{h}+\frac{M_{A} L_{g}\left(s_{m}^{\beta}+t_{m}^{\beta}\right)+M_{A}^{2} L_{g}\left(s_{m-1}^{\beta}+t_{m-1}^{\beta}\right)+\ldots+M_{A}^{m} L_{g}\left(s_{1}^{\beta}+t_{1}^{\beta}\right)}{\Gamma(\beta+1)} ;\right. \\
& \left.\frac{\left[M_{A} T^{\alpha}+M_{A}^{2}\left(t_{m}^{\alpha}+s_{m}^{\alpha}\right)+\ldots+M_{A}^{m+1}\left(t_{1}^{\alpha}+s_{1}^{\alpha}\right)\right]}{\Gamma(\alpha+1)}\left(D\left(1+B^{*}+F^{*}\right)+D^{\prime} q^{*}\right)\right\}<1 .
\end{aligned}
$$

Then the problem (1.2) has at least one mild solution.

Proof. We introduce the composition $Q=Q_{1}+Q_{2}$ where :

$$
\begin{aligned}
& Q_{1} x(t)=\left\{\begin{array}{l}
P_{\alpha}(t)\left(x_{0}+h(x)\right)+\int_{0}^{t}(t-s)^{\alpha-1} Q_{\alpha}(t-s) C(s) u(s) d s, t \in\left[0, t_{1}\right], \\
d_{i 1 x}+\frac{1}{\Gamma(\beta)} \int_{0}^{t}(t-s)^{\beta-1} g_{i}(s, x(s)) d s, t \in\left(t_{i}, s_{i}\right], i=1,2, . ., m, \\
P_{\alpha}\left(t-s_{i}\right) K_{i 1 x}+\int_{0}^{t}(t-s)^{\alpha-1} Q_{\alpha}(t-s) C(s) u(s) d s, t \in\left(s_{i}, t_{i+1}\right], i=1,2, . ., m,
\end{array}\right. \\
& Q_{2} x(t)=\left\{\begin{array}{l}
\int_{0}^{t}(t-s)^{\alpha-1} Q_{\alpha}(t-s)\left[f(s, x(s), F x(s), B x(s))+\int_{0}^{s} q(s-\tau) k(\tau, x(\tau)) d \tau\right] d s, t \in\left[0, t_{1}\right], \\
d_{i 2 x}, t \in\left(t_{i}, s_{i}\right], i=1,2, . ., m, \\
P_{\alpha}\left(t-s_{i}\right) K_{i 2 x}+\int_{0}^{t}(t-s)^{\alpha-1} Q_{\alpha}(t-s)\left[f(s, x(s), F x(s), B x(s))+\int_{0}^{s} q(s-\tau) k(\tau, x(\tau)) d \tau\right] d s, \\
t \in\left(s_{i}, t_{i+1}\right], i=1,2, . ., m,
\end{array}\right.
\end{aligned}
$$

with 


$$
\begin{aligned}
& \left\{\begin{array}{l}
d_{i 1 x}=P_{\alpha}\left(t_{i}-s_{i-1}\right) K_{(i-1) 1 x}+\int_{0}^{t_{i}}\left(t_{i}-s\right)^{\alpha-1} Q_{\alpha}\left(t_{i}-s\right) C(s) u(s) d s-\frac{1}{\Gamma(\beta)} \int_{0}^{t_{i}}\left(t_{i}-s\right)^{\beta-1} g_{i}(s, x(s)) d s, \\
i=1,2, . ., m, \\
K_{i 1 x}=d_{i 1 x}+\frac{1}{\Gamma(\beta)} \int_{0}^{s_{i}}\left(s_{i}-s\right)^{\beta-1} g_{i}(s, x(s)) d s-\int_{0}^{s_{i}}\left(s_{i}-s\right)^{\alpha-1} Q_{\alpha}\left(s_{i}-s\right) C(s) u(s) d s, i=1,2, . ., m, \\
K_{01 x}=x_{0}+h(x),
\end{array}\right. \\
& \left\{\begin{array}{l}
d_{i 2 x}=P_{\alpha}\left(t_{i}-s_{i-1}\right) K_{(i-1) 2 x}+\int_{0}^{t_{i}}\left(t_{i}-s\right)^{\alpha-1} Q_{\alpha}\left(t_{i}-s\right)[f(s, x(s), F x(s), B x(s))+ \\
\left.\int_{0}^{s} q(s-\tau) k(\tau, x(\tau)) d \tau\right] d s, i=1,2, . ., m, \\
K_{i 2 x}=d_{i 2 x}-\int_{0}^{s_{i}}\left(s_{i}-s\right)^{\alpha-1} Q_{\alpha}\left(s_{i}-s\right)\left[f(s, x(s), F x(s), B x(s))+\int_{0}^{s} q(s-\tau) k(\tau, x(\tau)) d \tau\right] d s, \\
i=1,2, . ., m, \\
K_{02 x}=0 .
\end{array}\right.
\end{aligned}
$$

Our proof will be divided into several steps.

- We show that $Q B_{r}(J) \subset B_{r}(J)$

where $B_{r}=\{x \in P C(J, X) ;\|x\| \leq r\}$ the ball with radius $r>0$,

$$
\begin{aligned}
& \text { and } K_{\alpha, \tau}=\alpha\left(\frac{1-\tau}{\alpha-\tau}\right)^{1-\tau}\|C u\|_{L^{1 / \tau}}, D^{\prime \prime}=D+D^{\prime} q^{*} \\
& \gamma_{1}=M_{A}^{m+1}\left[\left\|x_{0}\right\|+L_{h}^{\prime}\right]+\frac{M_{A}\left[D^{\prime \prime} T^{\alpha}+K_{\alpha, \tau} T^{\alpha-\tau}\right]}{\Gamma(\alpha+1)}, \\
& \gamma_{2}=\frac{M_{A}^{2}\left[D^{\prime \prime}\left(t_{m}^{\alpha}+s_{m}^{\alpha}\right)+K_{\alpha, \tau}\left(t_{m}^{\alpha-\tau}+s_{m}^{\alpha-\tau}\right)\right]+. .+M_{A}^{m+1}\left[D^{\prime \prime}\left(t_{1}^{\alpha}+s_{1}^{\alpha}\right)+K_{\alpha, \tau}\left(t_{1}^{\alpha-\tau}+s_{1}^{\alpha-\tau}\right)\right]}{\Gamma(\alpha+1)}, \\
& \gamma_{3}=\frac{M_{A} L_{g}^{\prime}\left(t_{i}^{\beta}+s_{m}^{\beta}\right)+M_{A}^{2} L_{g}^{\prime}\left(t_{m-1}^{\beta}+s_{m-1}^{\beta}\right)+\ldots+M_{A}^{m} L_{g}^{\prime}\left(t_{1}^{\beta}+s_{1}^{\beta}\right)}{\Gamma(\beta+1)},
\end{aligned}
$$

$$
\gamma_{4}=\frac{\left[M_{A} T^{\alpha}+M_{A}^{2}\left(t_{m}^{\alpha}+s_{m}^{\alpha}\right)+\ldots+M_{A}^{m+1}\left(t_{1}^{\alpha}+s_{1}^{\alpha}\right)\right]}{\Gamma(\alpha+1)}\left(D\left(1+B^{*}+F^{*}\right)+D^{\prime} q^{*}\right) .
$$

Here $\quad \frac{\gamma_{1}+\gamma_{2}+\gamma_{3}}{1-\gamma_{4}}<r$.

For any $x \in B_{r}$, we have:

Case1. For $t \in\left[0, t_{1}\right]$,

$$
\begin{aligned}
\|Q x(t)\| \leq & M_{A}\left[\left\|x_{0}\right\|+L_{h}^{\prime}\right]+\frac{M_{A} t_{1}^{\alpha}}{\Gamma(\alpha+1)}\left[D\left(1+r\left(1+B^{*}+F^{*}\right)\right)+D^{\prime} q^{*}(1+r)\right]+ \\
& \frac{K_{\alpha, \tau} t_{1}^{\alpha-\tau}}{\Gamma(\alpha+1)} M_{A} \\
\leq & M_{A}\left[\left\|x_{0}\right\|+L_{h}^{\prime}+\frac{D^{\prime \prime} t_{1}^{\alpha}+K_{\alpha, \tau} t_{1}^{\alpha-\tau}}{\Gamma(\alpha+1)}\right]+M_{A} \frac{\left(D\left(1+B^{*}+F^{*}\right)+D^{\prime} q^{*}\right) t_{1}^{\alpha}}{\Gamma(\alpha+1)} r \\
\leq & r .
\end{aligned}
$$

Similar to the proof of Theorem(4.1), we prove that: 
Case 2. For $t \in\left(t_{i}, s_{i}\right], i=1,2, \ldots, m$,

$$
\begin{aligned}
\|Q x(t)\| \leq & M_{A}^{i}\left[\left\|x_{0}\right\|+L_{h}^{\prime}\right]+\frac{M_{A}\left[D^{\prime \prime} t_{i}^{\alpha}+K_{\alpha, \tau} t_{i}^{\alpha-\tau}\right]}{\Gamma(\alpha+1)}+ \\
& \frac{M_{A}^{2}\left[D^{\prime \prime}\left(t_{i-1}^{\alpha}+s_{i-1}^{\alpha}\right)+K_{\alpha, \tau}\left(t_{i-1}^{\alpha-\tau}+s_{i-1}^{\alpha-\tau}\right)\right]+. .+M_{A}^{i}\left[D^{\prime \prime}\left(t_{1}^{\alpha}+s_{1}^{\alpha}\right)+K_{\alpha, \tau}\left(t_{1}^{\alpha-\tau}+s_{1}^{\alpha-\tau}\right)\right]}{\Gamma(\alpha+1)} \\
& +\frac{L_{g}^{\prime}\left(t_{i}^{\beta}+s_{i}^{\beta}\right)+M_{A} L_{g}^{\prime}\left(t_{i-1}^{\beta}+s_{i-1}^{\beta}\right)+\ldots+M_{A}^{i-1} L_{g}^{\prime}\left(t_{1}^{\beta}+s_{1}^{\beta}\right)}{\Gamma(\beta+1)} \\
& +\frac{\left[M_{A} t_{i}^{\alpha}+M_{A}^{2}\left(t_{i-1}^{\alpha}+s_{i-1}^{\alpha}\right)+M_{A}^{3}\left(t_{i-1}^{\alpha}+s_{i-1}^{\alpha}\right)+\ldots+M_{A}^{i}\left(t_{1}^{\alpha}+s_{1}^{\alpha}\right)\right]}{\Gamma(\alpha+1)} \times \\
& \left.\left(D\left(1+B^{*}+F^{*}\right)+D^{\prime} q^{*}\right)\right) r \leq r .
\end{aligned}
$$

Case 3. For $t \in\left(s_{i}, t_{i+1}\right]$,

$$
\begin{aligned}
\|Q x(t)\| \leq & M_{A}^{i+1}\left[\left\|x_{0}\right\|+L_{h}^{\prime}\right]+\frac{M_{A}\left[D^{\prime \prime} t_{i+1}^{\alpha}+K_{\alpha, \tau} t_{i+1}^{\alpha-\tau}\right]}{\Gamma(\alpha+1)} \\
& +\frac{M_{A}^{2}\left[D^{\prime \prime}\left(t_{i}^{\alpha}+s_{i}^{\alpha}\right)+K_{\alpha, \tau}\left(t_{i}^{\alpha-\tau}+s_{i}^{\alpha-\tau}\right)\right]+\ldots+M_{A}^{i+1}\left[D^{\prime \prime}\left(t_{1}^{\alpha}+s_{1}^{\alpha}\right)+K_{\alpha, \tau}\left(t_{1}^{\alpha-\tau}+s_{1}^{\alpha-\tau}\right)\right]}{\Gamma(\alpha+1)}+ \\
& +\frac{M_{A} L_{g}^{\prime}\left(t_{i}^{\beta}+s_{i}^{\beta}\right)+M_{A}^{2} L_{g}^{\prime}\left(t_{i-1}^{\beta}+s_{i-1}^{\beta}\right)+\ldots+M_{A}^{i} L_{g}^{\prime}\left(t_{1}^{\beta}+s_{1}^{\beta}\right)}{\Gamma(\beta+1)}+ \\
& +\frac{\left[M_{A} t_{i+1}^{\alpha}+M_{A}^{2}\left(t_{i}^{\alpha}+s_{i}^{\alpha}\right)+\ldots+M_{A}^{i+1}\left(t_{1}^{\alpha}+s_{1}^{\alpha}\right)\right]}{\Gamma(\alpha+1)}\left(D\left(1+B^{*}+F^{*}\right)+D^{\prime} q^{*}\right) r \leq r
\end{aligned}
$$

- $Q_{1}$ is a contraction on $B_{r}$. Let $x, y \in B_{r}$

Case 1. For $t \in\left[0, t_{1}\right]$, we have:

$\left\|Q_{1} x(t)-Q_{1} y(t)\right\| \leq M_{A} L_{h}\|x-y\|_{P C}<\|x-y\|_{P C}$.

Similar to the proof of Theorem(4.1), we prove that:

Case 2. For $t \in\left[t_{i}, s_{i}\right], 1 \leq i \leq m$,

$\left\|Q_{1} x(t)-Q_{1} y(t)\right\| \leq\left[M_{A}^{i} L_{h}+\frac{L_{g}\left(s_{i}^{\beta}+t_{i}^{\beta}\right)+M_{A} L_{g}\left(s_{i-1}^{\beta}+t_{i-1}^{\beta}\right)+\ldots+M_{A}^{i-1} L_{g}\left(s_{1}^{\beta}+t_{1}^{\beta}\right)}{\Gamma(\beta+1)}\right] \|$

$x-y \|_{P C}$

$<\|x-y\|_{P C}$.

For $t \in\left(s_{i}, t_{i+1}\right], 1 \leq i \leq m$,

$\left\|Q_{1} x(t)-Q_{1} y(t)\right\| \leq\left[M_{A}^{i+1} L_{h}+\frac{M_{A} L_{g}\left(s_{i}^{\beta}+t_{i}^{\beta}\right)+M_{A}^{2} L_{g}\left(s_{i-1}^{\beta}+t_{i-1}^{\beta}\right)+\ldots+M_{A}^{i} L_{g}\left(s_{1}^{\beta}+t_{1}^{\beta}\right)}{\Gamma(\beta+1)}\right] \|$

$x-y \|_{P C}$

$<\|x-y\|_{P C}$.

This implies that $Q_{1}$ is a contraction.

- $Q_{2}$ is continuous.

Let $\left(x_{n}\right)_{n \geq 0}$ be a sequence such that $\lim _{x \rightarrow \infty}\left\|x_{n}-x\right\|_{P C}=0$, we have :

Case 1. For $t \in\left[0, t_{1}\right]$

$$
\begin{aligned}
\left\|Q_{2} x_{n}(t)-Q_{2} x(t)\right\| \leq & \| \int_{0}^{t}(t-s)^{\alpha-1} Q_{\alpha}(t-s)\left(\left[f\left(s, x_{n}(s), F x_{n}(s), B x_{n}(s)\right)+\right.\right. \\
& \int_{0}^{s} q(s-\tau) k\left(\tau, x_{n}(\tau)\right) d \tau-f(s, x(s), F x(s), B x(s))- \\
& \left.\left.\int_{0}^{s} q(s-\tau) k(\tau, x(\tau)) d \tau\right)\right] d s \| \\
\leq & \frac{M_{A} t_{1}^{\alpha}}{\Gamma(\alpha+1)}\left(\left\|f\left(., x_{n}(.), F x_{n}(.), B x_{n}(.)\right)-f(., x(.), F x(.), B x(.))\right\|_{P C}+\right. \\
& \left.+\left\|q^{*}\left[k\left(., x_{n}(.)\right)-k(., x(.))\right]\right\|_{P C}\right) .
\end{aligned}
$$


Similar to the proof of Theorem(4.1), we prove that:

Case 2. For $t \in\left(t_{i}, s_{i}\right], i=1,2, \ldots, m$,

$$
\begin{aligned}
\left\|Q_{2} x_{n}(t)-Q_{2} x(t)\right\| \leq & {\left[\frac{M_{A} t_{i}^{\alpha}+M_{A}^{2}\left(t_{i-1}^{\alpha}+s_{i-1}^{\alpha}\right)+\ldots+M_{A}^{i}\left(t_{1}^{\alpha}+s_{1}^{\alpha}\right)}{\Gamma(\alpha+1)}\right] \times } \\
& \left(\left\|f\left(., x_{n}(.), F x_{n}(.),, B x_{n}(.)\right)-f(., x(.), F x(.), B x(.))\right\|_{P C}+\right. \\
& \left.\left\|q^{*}\left[k\left(., x_{n}(.)\right)-k(., x(.))\right]\right\|_{P C}\right) .
\end{aligned}
$$

Case 3. For $t \in\left(s_{i}, t_{i+1}\right]$,

$$
\begin{aligned}
\left\|Q_{2} x_{n}(t)-Q_{2} x(t)\right\| \leq & {\left[\frac{M_{A} t_{i+1}^{\alpha}+M_{A}^{2}\left(t_{i}^{\alpha}+s_{i}^{\alpha}\right)+\ldots+M_{A}^{i+1}\left(t_{1}^{\alpha}+s_{1}^{\alpha}\right)}{\Gamma(\alpha+1)}\right] \times } \\
& \times\left(\left\|f\left(., x_{n}(.), F x_{n}(.), B x_{n}(.)\right)-f(., x(.), F x(.), B x(.))\right\|_{P C}+\right. \\
& \left.\left\|q^{*}\left[k\left(., x_{n}(.)\right)-k(., x(.))\right]\right\|_{P C}\right) .
\end{aligned}
$$

- $Q_{2}$ is compact.

1. We have $Q_{2} B_{r} \subseteq B_{r}$, then $Q_{2}$ is uniformly bounded on $B_{r}$,

2. For $x \in B_{r}$, we have the following:

for $0 \leq t^{\prime}<t^{\prime \prime} \leq t_{1}$, we have:

$$
\begin{aligned}
& \left\|Q_{2} x\left(t^{\prime \prime}\right)-Q_{2} x\left(t^{\prime}\right)\right\| \leq \| \int_{0}^{t^{\prime \prime}}\left(t^{\prime \prime}-s\right)^{\alpha-1} Q_{\alpha}\left(t^{\prime \prime}-s\right)(f(s, x(s), F x(s), B x(s))+ \\
& \left.\int_{0}^{s} q(s-\tau) k(\tau, x(\tau)) d \tau\right) d s-\int_{0}^{t^{\prime}}\left(t^{\prime}-s\right)^{\alpha-1} Q_{\alpha}\left(t^{\prime}-s\right) \times \\
& \left(f(s, x(s), F x(s), B x(s))+\int_{0}^{s} q(s-\tau) k(\tau, x(\tau)) d \tau\right) d s \| \\
& \leq I_{1}+I_{2}+I_{3} \text {, } \\
& I_{1}=\left\|\int_{t^{\prime}}^{t^{\prime \prime}}\left(t^{\prime \prime}-s\right)^{\alpha-1} Q_{\alpha}\left(t^{\prime \prime}-s\right)\left(f(s, x(s), F x(s), B x(s))+\int_{0}^{s} q(s-\tau) k(\tau, x(\tau)) d \tau\right) d s\right\| \\
& I_{2}=\| \int_{0}^{t^{\prime}}\left(t^{\prime}-s\right)^{\alpha-1}\left[Q_{\alpha}\left(t^{\prime \prime}-s\right)-Q_{\alpha}\left(t^{\prime}-s\right)\right](f(s, x(s), F x(s), B x(s))+ \\
& \left.\int_{0}^{s} q(s-\tau) k(\tau, x(\tau)) d \tau\right) d s \| \\
& I_{3}=\| \int_{0}^{t^{\prime}}\left[\left(t^{\prime \prime}-s\right)^{\alpha-1}-\left(t^{\prime}-s\right)^{\alpha-1}\right] Q_{\alpha}\left(t^{\prime \prime}-s\right)(f(s, x(s), F x(s), B x(s))+ \\
& \left.\int_{0}^{s} q(s-\tau) k(\tau, x(\tau)) d \tau\right) d s \| \text {. } \\
& I_{1} \leq \frac{\alpha M_{A}\left[D\left(1+r\left(1+B^{*}+F^{*}\right)\right)+D^{\prime} q^{*}(1+r)\right]}{\Gamma(\alpha+1)} \int_{t^{\prime}}^{t^{\prime \prime}}\left(t^{\prime \prime}-s\right)^{\alpha-1} d s \\
& \leq \frac{M_{A}\left[D\left(1+r\left(1+B^{*}+F^{*}\right)\right)+D^{\prime} q^{*}(1+r)\right]}{\Gamma(\alpha+1)}\left(t^{\prime \prime}-t^{\prime}\right)^{\alpha} \longrightarrow 0, t^{\prime \prime}-t^{\prime} \longrightarrow 0 . \\
& I_{2} \leq\left[D\left(1+r\left(1+B^{*}+F^{*}\right)\right)+D^{\prime} q^{*}(1+r)\right] \int_{0}^{t^{\prime}}\left(t^{\prime}-s\right)^{\alpha-1}\left\|Q_{\alpha}\left(t^{\prime \prime}-s\right)^{\alpha-1}-Q_{\alpha}\left(t^{\prime}-s\right)^{\alpha-1}\right\| d s \longrightarrow 0, t^{\prime \prime}-t^{\prime} \longrightarrow 0 \text {. }
\end{aligned}
$$




$$
\begin{aligned}
& I_{3} \leq \frac{M_{A}\left[D\left(1+r\left(1+B^{*}+F^{*}\right)\right)+D^{\prime} q^{*}(1+r)\right]}{\Gamma(\alpha)} \int_{0}^{t^{\prime}}\left(\left(t^{\prime \prime}-s\right)^{\alpha-1}-\left(t^{\prime}-s\right)^{\alpha-1}\right) d s \\
& \leq \frac{M_{A}\left[D\left(1+r\left(1+B^{*}+F^{*}\right)\right)+D^{\prime} q^{*}(1+r)\right]}{\Gamma(\alpha+1)}\left(t^{\prime \prime}-t^{\prime}\right)^{\alpha} \longrightarrow 0 ; t^{\prime \prime}-t^{\prime} \longrightarrow 0 .
\end{aligned}
$$

Case 1. For $t_{i} \leq t^{\prime}<t^{\prime \prime} \leq s_{i}$,

$\left\|Q_{2} x\left(t^{\prime \prime}\right)-Q_{2} x\left(t^{\prime}\right)\right\|=0$.

Case 2. For $s_{i} \leq t^{\prime}<t^{\prime \prime} \leq t_{i+1}$,

$$
\left\|Q_{2} x\left(t^{\prime \prime}\right)-Q_{2} x\left(t^{\prime}\right)\right\| \leq I_{1}+I_{2}+I_{3}+\left\|\left(P_{\alpha}\left(t^{\prime \prime}-s_{i}\right)-P_{\alpha}\left(t^{\prime}-s_{i}\right)\right) K_{i 2 x}\right\| .
$$

From $\left(H_{1}\right)$ and the proof of lemma 3.4 in [14] we have that the continuity of $P_{\alpha}(t)$ and $Q_{\alpha}(t)(t>0)$ in $\mathrm{t}$ is in the uniform operator topology, we deduce that the right-hand side of (4.1) tends to 0 independently of $x \in B_{r}$ as $t^{\prime \prime} \rightarrow t^{\prime}$.

Case 3. For $t_{i} \leq t^{\prime}<s_{i}<t^{\prime \prime} \leq t_{i+1}$,

$$
\begin{aligned}
\left\|Q_{2} x\left(t^{\prime \prime}\right)-Q_{2} x\left(t^{\prime}\right)\right\| \leq & \| P_{\alpha}\left(t^{\prime \prime}-s_{i}\right) K_{i 2 x}+\int_{0}^{t^{\prime \prime}}\left(t^{\prime \prime}-s\right)^{\alpha-1} Q_{\alpha}\left(t^{\prime \prime}-s\right)(f(s, x(s), F x(s), B x(s))+ \\
& \left.+\int_{0}^{s} q(s-\tau) k(\tau, x(\tau)) d \tau\right) d s-d_{i 2 x} \| \longrightarrow 0
\end{aligned}
$$

indenpendently of $x \in B_{r}$, as $t^{\prime \prime} \longrightarrow t^{\prime}$ we have $\left(t^{\prime \prime} \longrightarrow s_{i}\right)$.

In conclusion, $\left\|Q_{2} x\left(t^{\prime \prime}\right)-Q_{2} x\left(t^{\prime}\right)\right\| \longrightarrow 0$, as $t^{\prime \prime}-t^{\prime} \longrightarrow 0$, which implies that $Q_{2}\left(B_{r}(J)\right)$ is equicontinuous .

We have $Q_{2} B_{r} \subseteq B_{r}$, let $Q_{2} B_{r}(t)=\left\{Q_{2} x(t) ; x \in B_{r}\right\}$ for $t \in J$.

3. $Q_{2} B_{r}(t)$ is relatively compact.

$Q_{2} B_{r}(0)=\{0\}$ is compact.

For $t \in\left[0, t_{1}\right]$.

For each $\epsilon \in(0, t)$ and $\delta>0$, we define a set:

$Q_{2}^{\epsilon, \delta}\left(B_{r}\right)(t)=\left\{Q_{2}^{\epsilon, \delta} x(t) ; x \in B_{r}\right\}$

with

$$
\begin{aligned}
Q_{2}^{\epsilon, \delta} x(t)= & \alpha \int_{0}^{t-\epsilon} \int_{\delta}^{\infty} \theta(t-s)^{\alpha-1} \xi_{\alpha}(\theta) T\left((t-s)^{\alpha} \theta\right)(f(s, x(s), F x(s), B x(s))+ \\
& \left.\int_{0}^{s} q(s-\tau) k(\tau, x(\tau)) d \tau\right) d \theta d s \\
= & \alpha \int_{0}^{t-\epsilon} \int_{\delta}^{\infty} \theta(t-s)^{\alpha-1} \xi_{\alpha}(\theta)\left[T\left(\epsilon^{\alpha} \delta\right) T\left((t-s)^{\alpha} \theta-\epsilon^{\alpha} \delta\right)\right](f(s, x(s), F x(s), B x(s))+ \\
& \left.\int_{0}^{s} q(s-\tau) k(\tau, x(\tau)) d \tau\right) d \theta d s \\
= & \alpha T\left(\epsilon^{\alpha} \delta\right) \int_{0}^{t-\epsilon} \int_{\delta}^{\infty} \theta(t-s)^{\alpha-1} \xi_{\alpha}(\theta) T\left((t-s)^{\alpha} \theta-\epsilon^{\alpha} \delta\right)(f(s, x(s), F x(s), B x(s))+ \\
& \left.\int_{0}^{s} q(s-\tau) k(\tau, x(\tau)) d \tau\right) d \theta d s .
\end{aligned}
$$

(Observe that $\theta \geq \delta$ and $t-\epsilon \geq s$, hence $(t-s)^{\alpha} \theta-\epsilon^{\alpha} \delta \geq 0$ ) Since the operator $T\left(\epsilon^{\alpha} \delta\right)\left(\epsilon^{\alpha} \delta>0\right)$ is compact, the set $Q_{2}^{\epsilon, \delta} B_{r}(t)$ is relatively compact in X. Moreover, for every $x \in B_{r}$ we have 


$$
\begin{aligned}
\left\|Q_{2} x(t)-Q_{2}^{\epsilon, \delta} x(t)\right\| \leq & \alpha \| \int_{0}^{t} \int_{0}^{\delta} \theta(t-s)^{\alpha-1} \xi_{\alpha}(\theta) T\left((t-s)^{\alpha} \theta\right)(f(s, x(s), F x(s), B x(s))+ \\
& \left.+\int_{0}^{s} q(s-\tau) k(\tau, x(\tau)) d \tau\right) d \theta d s+\int_{0}^{t} \int_{\delta}^{\infty} \theta(t-s)^{\alpha-1} \xi_{\alpha}(\theta) T\left((t-s)^{\alpha} \theta\right) \times \\
& \times\left(f(s, x(s), F x(s), B x(s))+\int_{0}^{s} q(s-\tau) k(\tau, x(\tau)) d \tau\right) d \theta d s \\
& -\int_{0}^{t-\epsilon} \int_{\delta}^{\infty} \theta(t-s)^{\alpha-1} \xi_{\alpha}(\theta) T\left((t-s)^{\alpha} \theta\right)(f(s, x(s), F x(s), B x(s))+ \\
& \left.\int_{0}^{s} q(s-\tau) k(\tau, x(\tau)) d \tau\right) d \theta d s \| \\
\leq & G_{1}+G_{2} .
\end{aligned}
$$

With

$G_{1}=\alpha\left\|\int_{0}^{t} \int_{0}^{\delta} \theta(t-s)^{\alpha-1} \xi_{\alpha}(\theta) T\left((t-s)^{\alpha} \theta\right)\left(f(s, x(s), F x(s), B x(s))+\int_{0}^{s} q(s-\tau) k(\tau, x(\tau)) d \tau\right) d \theta d s\right\|$

and

$G_{2}=\alpha\left\|\int_{t-\epsilon}^{t} \int_{\delta}^{\infty} \theta(t-s)^{\alpha-1} \xi_{\alpha}(\theta) T\left((t-s)^{\alpha} \theta\right)\left(f(s, x(s), F x(s), B x(s))+\int_{0}^{s} q(s-\tau) k(\tau, x(\tau)) d \tau\right) d \theta d s\right\|$.

We have

$$
\begin{aligned}
G_{1} & \leq \alpha M_{A} \int_{0}^{t}(t-s)^{\alpha-1}\left\|\left(f(s, x(s), F x(s), B x(s))+\int_{0}^{s} q(s-\tau) k(\tau, x(\tau)) d \tau\right)\right\| d s \int_{0}^{\delta} \theta \xi_{\alpha}(\theta) d \theta \\
& \leq M_{A} t_{1}^{\alpha}\left[D\left(1+r\left(1+B^{*}+F^{*}\right)\right)+D^{\prime} q^{*}(1+r)\right] \int_{0}^{\delta} \theta \xi_{\alpha}(\theta) d \theta,
\end{aligned}
$$

and

$$
\begin{aligned}
G_{2} & \leq \alpha M_{A} \int_{t-\epsilon}^{t}(t-s)^{\alpha-1}\left\|\left(f(s, x(s), F x(s), B x(s))+\int_{0}^{s} q(s-\tau) k(\tau, x(\tau)) d \tau\right)\right\| d s \int_{\delta}^{\infty} \theta \xi_{\alpha}(\theta) d \theta \\
& \leq M_{A} \epsilon^{\alpha}\left[D\left(1+r\left(1+B^{*}+F^{*}\right)\right)+D^{\prime} q^{*}(1+r)\right] \int_{0}^{\infty} \theta \xi_{\alpha}(\theta) d \theta \\
& \leq \frac{M_{A}\left[D\left(1+r\left(1+B^{*}+F^{*}\right)\right)+D^{\prime} q^{*}(1+r)\right]}{\Gamma(\alpha+1)} \epsilon^{\alpha}
\end{aligned}
$$

Then $\|\left(Q_{2} x(t)-Q_{2}^{\epsilon, \delta} x(t) \| \longrightarrow 0\right.$, as $\epsilon \longrightarrow 0 ; \delta \longrightarrow 0$

This means that there are relatively compact sets arbitrarily close to the set $Q_{2} B_{r}(t)$. Hence the set $Q_{2} B_{r}(t)$ is also relatively compact in $X$.

For $t_{i}<t \leq s_{i}, i=1,2, \ldots, m$, in such case

$Q_{2} B_{r}(t)=\left\{d_{i 2 x}, x \in B_{r}\right\}$ is compact.

For $s_{i}<t \leq t_{i+1}, i=1,2, \ldots, m$, $Q_{2} B_{r}(t)=\left\{P_{\alpha}\left(t-s_{i}\right) K_{i 2 x}+\int_{0}^{t}(t-s)^{\alpha-1} Q_{\alpha}(t-s)\left(f(s, x(s), F x(s), B x(s))+\int_{0}^{s} q(s-\tau) k(\tau, x(\tau)) d \tau\right) d s, x \in B_{r}\right\}$. By the same argument in case $1\left(t \in\left[0, t_{1}\right]\right)$ and $P_{\alpha}\left(t-s_{i}\right)$ is a compact operator, we know that $Q_{2} B_{r}(t)$ is relatively compact.

\section{Example}

In this section, we give two examples which illustrate the applicability of our results. 
Throughout this section, we let $X=L^{2}(0,1), J=[0,1], t_{0}=s_{0}=0, t_{1}=\frac{1}{3}$, $s_{1}=\frac{2}{3}, T=1$.

Define $A x=\frac{\partial^{2}}{\partial^{2} v} x$ for $x \in D(A)=\left\{x \in X: \frac{\partial x}{\partial v}, \frac{\partial^{2} x}{\partial^{2} v} \in X, x(0)=x(1)=0\right\}$.

Then $A$ is the infinitesimal generator of strongly continuous semigroup $\{T(t), t \geq 0\}$ on $X$. In addition $T(t)$ is compact and $\|T(t)\| \leq 1=M_{A}$, for all $t \geq 0$. See [10]

Example 4.3. Consider

$$
\left\{\begin{array}{l}
\frac{\partial^{\alpha}}{\partial t^{\alpha}} y(t, v)=\frac{\partial^{2}}{\partial v^{2}} y(t, v)+\frac{1}{24} \sin \left[y(t, v)+\int_{0}^{t} \frac{e^{-(s-t)}}{80} y(s, v)+\int_{0}^{t} \frac{e^{-2(s-t)}}{160} y(s, v) d s\right]+ \\
\int_{0}^{t} e^{-(s-t)} \frac{1}{24} \cos (y(s, v)) d s+C(t, v), v \in(0,1), t \in\left[0, \frac{1}{3}\right] \cup\left(\frac{2}{3}, 1\right], \\
\frac{\partial^{\beta}}{\partial t^{\beta}} y(t, v)=\frac{1}{8} \cos (y(t, v)), t \in\left(\frac{1}{3}, \frac{2}{3}\right], \\
y(t, v)=y_{0}+\frac{1}{8 e^{t}}\left(y\left(s_{1}, v\right)+y\left(t_{1}, v\right)\right) .
\end{array}\right.
$$

Denote $x(t)(v)=y(t, v)$ and $C(t, v)=C(t) u(t)(v)$

This problem can be abstracted into;

$(P)\left\{\begin{array}{l}{ }^{c} D^{\alpha} x(t)(v)=A x(t)(v)+f(t, x(t), F x(t), B x(t))(v)+\int_{0}^{t} q(t-s) k(s, x(s)(v)) d s+C(t) u(t)(v) \\ t \in\left[0, \frac{1}{3}\right] \cup\left(\frac{2}{3}, 1\right], u \in U_{a d}, \\ { }^{c} D^{\beta} x(t)=g_{1}(t, x(t)), t \in\left(t_{i}, s_{i}\right], t \in\left(\frac{1}{3}, \frac{2}{3}\right], \\ x(0)=x_{0}+h(x) .\end{array}\right.$

Where: $B x(t)(v)=\int_{0}^{t} \frac{e^{-2(s-t)}}{160} y(s, v) d s$

$F x(t)(v)=\int_{0}^{t} \frac{e^{-(s-t)}}{80} y(s, v) d s, f(t, x(t), F x(t), B x(t))(v)=\frac{1}{24} \sin \left[y(t, v)+\int_{0}^{t} \frac{e^{-(s-t)}}{80} y(s, v)+\right.$ $\left.\int_{0}^{t} \frac{e^{-2(s-t)}}{160} y(s, v) d s\right]$

$k(t, x(t))(v)=\frac{1}{24} \cos (x(t)(v)), g_{1}(t, x(t))(v)=\frac{1}{8} \cos (x(t)(v)), h(t, x(t))(v)=$ $\frac{1}{8}\left(x\left(s_{1}\right)(v)+x\left(t_{1}\right)(v)\right)$, $q(t)=\frac{e^{t}}{4}$ and $\alpha=0.85, \beta=0.95$,

In this case we have: $L_{k}=L_{f}=C_{f}=M_{f}=\frac{1}{24}, L_{g}=L_{h}=\frac{1}{8}$, and $F^{*}=B^{*}=$ $\frac{1}{40}, q^{*}=\frac{e-1}{4},\left[L_{h}+\frac{1+\left(t_{1}^{\alpha}+s_{1}^{\alpha}\right)}{\Gamma(\alpha+1)}\left(L_{f}+q^{*} L_{k}+M_{f} B^{*}+C_{f} F^{*}\right)+\frac{L_{g}\left(t_{1}^{\beta}+s_{1}^{\beta}\right)}{\Gamma(\beta+1)}\right] \approx$ $0.393717<1$. 
Which implies that all the assumptions of theorem 4.1 are satisfied. Therefore, there exists a unique mild solution to our problem.

Example 4.4. Consider

$$
\left\{\begin{array}{l}
\frac{\partial^{\alpha}}{\partial t^{\alpha}} y(t, v)=\frac{\partial^{2}}{\partial v^{2}} y(t, v)+\frac{1}{24 e^{t}} \frac{|y(t, v)+B y(t, v)+F y(t, v)|}{1+|y(t, v)+B y(t, v)+F y(t, v)|}+\int_{0}^{t} \frac{1}{24 e^{-(t-s)}} \frac{e^{s}|y(s, v)|}{1+|y(s, v)|} d s+C(t, v) \\
v \in(0,1), t \in\left[0, \frac{1}{3}\right] \cup\left(\frac{2}{3}, 1\right] \\
\frac{\partial^{\beta}}{\partial t^{\beta}} y(t, v)=\frac{1}{8 e^{t}} \frac{|y(t, v)|}{1+|y(t, v)|}, t \in\left(\frac{1}{3}, \frac{2}{3}\right] \\
y(t, v)=y_{0}+\frac{1}{8 e^{t}}\left(y\left(s_{1}, v\right)+y\left(t_{1}, v\right)\right) .
\end{array}\right.
$$

Denote $x(t)(v)=y(t, v)$ and $C(t, v)=C(t) u(t)(v)$.

This problem can be abstracted into $(P)$. Where: $B x(t)(v)=\int_{0}^{t} \frac{e^{-2(s-t)}}{160} y(s, v) d s$

$$
\begin{aligned}
& F x(t)(v)=\int_{0}^{t} \frac{e^{-(s-t)}}{80} y(s, v) d s, f(t, x(t), F x(t), B x(t))(v)=\frac{1}{24 e^{t}} \frac{|x(t)(v)+B x(t)(v)+F x(t)(v)|}{1+|x(t)(v)+B x(t)(v)+F x(t)(v)|} \\
& \quad k(t, x(t))(v)=\frac{1}{24} \frac{e^{t}|y(t, v)|}{1+|y(t, v)|}, g_{1}(t, x(t))(v)=\frac{1}{8 e^{t}} \frac{|y(t, v)|}{1+|y(t, v)|}, h(t, x(t))(v)= \\
& \frac{1}{8 e^{t}}\left(x\left(s_{1}\right)(v)+x\left(t_{1}\right)(v)\right), \\
& q(t)=\frac{e^{t}}{4} \text { and } \alpha=0.75, \beta=0.65 .
\end{aligned}
$$

In this case we have: $L_{h}=L_{g}=\frac{1}{8}, D=D^{\prime}=\frac{1}{24}$, and $B^{*}=F^{*}=\frac{1}{40}$,

$$
\begin{aligned}
& q^{*}=\frac{e-1}{4} \\
& \quad L_{h}+L_{g} \frac{\left(t_{1}^{\beta}+s_{1}^{\beta}\right)}{\Gamma(\beta+1)} \approx 0.3 \\
& \quad \text { and } \frac{1+\left(t_{1}^{\alpha}+s_{1}^{\alpha}\right)}{\Gamma(\alpha+1)}\left(D\left(1+B^{*}+F^{*}\right)+D^{\prime} q^{*}\right) \approx 0.145993 .
\end{aligned}
$$

We have $\max \{0.3 ; 0.145993\}<1$.

This implies that all assumptions of theorem 4.2 are satisfied. Then, our problem has at least one mild solution.

\section{REFERENCES}

[1] A. Angaraj, K. Karthikeyan, Existence of solutions for impulsive neutral functional differential equations with non-local conditions, Nonlinear Anal. 70(2009), 2717-2721.

[2] A. Anguraj, M. Lathamaheshwari, Existence results for fractional differential equations with infinite delay and interval impulsive conditions, Malaya J.Mat 2(1)(2014) 16-23.

[3] X. Fu, X. Liu and B. Lu, On a new class of impulsive fractional evolution equations, Advances in difference equations (2015) 2015:227.

[4] H. James, Liu, Nonlinear impulsive evolution equations, Dynam. Contin. Discrete Impuls. Systems 6(1999),77-85.

[5] Hernandez, E., O'Regan, D.: On a new class of abstract impulsive differential equations. Proc. Am. Math. Soc. 141, 1641-1649(2013) 
[6] Kilbas, AA, Srivastava, HM, Trujillo, JJ:Theory and Applications of Fractional Differential Equations. North-Holland Mathematics Studies, vol. 204.Elsevier, Amesterdam(2006)

[7] V. Lakshmikantham, DD. Bainov, P.S. Simeonov, Theory of impulsive differential equations, World scientific, Singapore, 1989.

[8] Shengda Liu, JinRong Wang, Optimal Controls of Systems Governed by Semilinear Fractional Differential Equations with Not Instantaneous Impulses, J Optim Theory Appl. DOI 10.1007/s10957-017-1122-3.

[9] J.J. Nieto, D. O'Regan, Variational approach to impulsive differential equations, Nonlinear Anal, Real World Appl. 10(2009), 680-690.

[10] A. Pazy, Semigroups of Linear Operators and Applications to Partial Differential Equations, Springer, Berlin,1983.

[11] W. Wei, X. Xiang, Y. Peng:Nonlinear impulsive integro-differential equation of mixed type and optimal controls. Optimization 55, 141-156(2006).

[12] Y. Zhou, F. Jiao: Nonlocal Cauchy problem for fractional evolution equations. Nonlinear Anal, Real World Appl. 11, 4465-4475(2010)

[13] Peter L.Falb, Infinite Dimensional Control Problems:On the Closure of the Set of Attainable States for Linear Systems, Mathematical Analysis and Application 9,12-22(1964).

[14] Y. Zhou, F. Jiao:Existence of mild solutions for fractional neutral evolution equations. Comput. Math. Appl. 59, 1063-1077(2010)

(K. Hilal) Sultan Molay Sliman university, department of Mathematics , BP523 2300 Beni Mellal, Morocco

E-mail address, K. Hilal: hilal khalid@yahoo.fr

(L. Ibnelazyz) Sultan Molay Sliman university, Department of Mathematics , BP523 2300 Beni Mellal, Morocco

E-mail address, L. Ibnelazyz: ibelazyzlahcen@gmail.com

(K. Guida) Sultan Molay Sliman university, department of Mathematics, BP523 2300 Beni Mellal, Morocco

E-mail address, K. Guida: guida.karim@gmail.com

(S. Melliani) Sultan Molay Sliman university, Department of Mathematics, BP523 2300 Beni Mellal, Morocco

E-mail address, S. Melliani: saidmelliani@gmail.com 